\title{
Metabolism of Exogenously Administered Natural Surfactant in the Newborn Lamb
}

\author{
THEODORE GLATZ, ${ }^{(21)}$ MACHIKO IKEGAMI, AND ALAN JOBE \\ Fetal-Maternal Research Laboratories, Department of Pediatrics, Harbor-UCLA Medical Center, Torrance, \\ California, USA
}

\begin{abstract}
Summary
$\left[{ }^{3} \mathrm{H}\right]$-Palmitate labeled natural lamb surfactant and free $\left[{ }^{14} \mathrm{C}\right]-$ choline were mixed with the lung fluid of 11 term lambs at cesarean section, before the first breath. After receiving the isotope, the lambs were delivered, allowed to breathe spontaneously, and were subsequently sacrificed from $5 \mathrm{~min}$ to $96 \mathrm{~h}$ of age. Alveolar washes, lung homogenates, microsomal and lamellar body fractions of lungs, and pulmonary alveolar macrophages were examined for the presence of labeled phosphatidylcholine. Analysis of the labeled natural surfactant kinetic data revealed an apparent $t_{1 / 2}$ of phosphatidylcholine in the whole lung of 6.0 days. This half-life can be interpreted only as a rough estimate. Appearance of considerable $\left[{ }^{3} \mathrm{H}\right.$ ] labeled phosphatidylcholine in the lung homogenates demonstrated uptake of phosphatidylcholine from alveoli into lung tissue. The surfactant-associated label in homogenates was localized preferentially to lamellar body fractions. Some of the administered $\left[{ }^{14} \mathrm{C}\right]$-choline appeared in phosphatidylcholine. Almost all of this labeled phosphatidylcholine was associated with the homogenate. Extremely small \% of administered $\left[{ }^{3} \mathrm{H}\right]$ and $\left[{ }^{14} \mathrm{C}\right]$ were found in pulmonary alveolar macrophages.
\end{abstract}

\section{Speculation}

The prolonged $t_{1 / 2}$ measured in this study indicates very slow turnover of alveolar phosphatidylcholine and/or considerable reutilization of label and possibly of the entire phosphatidylcholine molecule. The concentration of label in lamellar bodies raises the possibility that alveolar phosphatidylcholine may be degraded and its components utilized for synthesis of new phosphatidylcholine or that alveolar phosphatidylcholine may be absorbed into type-2 alveolar cells and resecreted intact. The finding of $\left[{ }^{14} \mathrm{C}\right]$ labeled phosphatidylcholine in lung homogenate but not in alveolar wash indicates that, even if choline were released by intra-alveolar degradation of surfactant, it is not used to a significant degree for synthesis of new surfactant phosphatidylcholine.

The ultimate fate of surfactant phospholipids, once secreted into the alveoli, remains to be defined. Reasonable speculations include: (1) removal via the lymphatic or circulatory systems, (2) metabolism by alveolar macrophages, (3) surface spreading into the airways, or (4) reuptake into pulmonary parenchymal cells with reutilization of all or part of the phospholipid molecule. Estimates of the biologic half-life $\left(\mathrm{t}_{1 / 2}\right)$ of surfactant phospholipids in lung parenchyma and in the alveoli have been derived by utilizing radiolabeled phospholipid precursors. The estimated half-life values vary, depending on type of labeled phospholipid precursor used, type of animal studied, and the maturity of the animals. An approximate $t_{1 / 2}$ of $16 \mathrm{~h}$ has been reported for lung phosphatidylcholine (PC) in adult rats and rabbits, using radiolabeled palmitic acid as a precursor $(10,11,19,20)$. Again using labeled palmitic acid, the biologic $t_{1 / 2}$ for alveolar surfactant was estimated to be 54 and $45 \mathrm{~h}$ in newborn and adult sheep, respectively (8). A common concern with these studies is that the reported $t_{1 / 2}$ may be in error due to continued secretion of isotope into the alveoli as the surfactant decay curve is being observed, which tends to give a prolonged $t_{1 / 2}$. Any reabsorption and reutilization of the alveolar surfactant by lung tissue would further complicate the estimation of $a_{t_{1 / 2}}$ for alveolar surfactant.

The present study focuses primarily on the length of survival of surfactant in newborn animals, once it is in the airways. In addition, we examined the possibility of reuptake of surfactant or its precursors from the alveoli into the lung tissue, as well as the role of the pulmonary alveolar macrophages in the breakdown of alveolar surfactant.

\section{MATERIALS AND METHODS}

Animals. Eleven western mix bred lambs were delivered by cesarean section from date-mated ewes at $146 \pm 0.5$ (S.E.) days gestation (term for singletons in this species is 150 days). Six of the lambs resulted from twin pregnancies. Initially, only the heads of the newborns were delivered, and $5.0 \mathrm{~mm}$ internal diameter cuffed endotracheal tubes were inserted into the tracheas and 10 $\mathrm{ml}$ of tracheal fluid was removed. Before the first breath, a mixture of about $2 \mu \mathrm{Ci} \mathrm{[}{ }^{3} \mathrm{H}$ ]-palmitate labeled natural surfactant (containing $105 \pm 14 \mu$ moles phosphatidylcholine) and $2 \mu \mathrm{Ci}\left[{ }^{14} \mathrm{C}\right]$-choline was diluted to a total volume of $20 \mathrm{ml}$ with normal saline and injected by syringe into the endotracheal tube of each newborn. The isotope suspension plus tracheal fluid was flushed in and out of the syringe four to five times to mix the isotope with the residual fetal lung fluid. The endotracheal tube was clamped to prevent loss of the isotope and the cesarean section was completed.

Umbilical venous blood was drawn from all animals for blood gas and $\mathrm{pH}$ analysis. When the umbilical cords were cut, the endotracheal tubes were unclamped and the animals were hand ventilated with $100 \%$ oxygen at a peak inspiratory pressure of 20 $\mathrm{cm} \mathrm{H}_{2} \mathrm{O}$ with a $500 \mathrm{ml}$ anesthesia bag for $5 \mathrm{~min}$. All animals began breathing spontaneously when the umbilical cord was cut. Those animals observed for longer than $6 \mathrm{~h}$ were bottle fed with standard infant formula. Lambs were sacrificed periodically, from $5 \mathrm{~min}$ to $96 \mathrm{~h}$ after birth, with a rapid intravenous injection of $1.0 \mathrm{~g}$ of pentobarbital (Euthanol-6). The animals were dried (if wet) and weighed at sacrifice.

Isotopes. $\left[{ }^{3} \mathrm{H}\right]$-Palmitate labeled natural surfactant was obtained from healthy newborn lambs. Approximately $35 \mathrm{~h}$ before sacrifice, two to seven-day-old lambs were given an intravenous injection of $15 \mathrm{mCi}\left[9,10-{ }^{3} \mathrm{H}\right]$-palmitic acid (New England Nuclear-17.6 $\mathrm{Ci} / \mathrm{mmole}$ ) in a $4 \%$ bovine serum albumin-physiologic saline solution. The lungs were removed intact and lavaged four times to yield a wash volume of about 1 liter. Surfactant was recovered from the lavage fluid by centrifugation (12). Of the label recovered in the surfactant, $69.8 \%$ was in phosphatidylcholine. The surfactant is highly surface active and effective as therapy for respiratory distress in premature lambs $(12)$. $\left[{ }^{14} \mathrm{C}\right]$-choline $(54 \mathrm{Ci} / \mathrm{mole})$ in ethanol was obtained from New England Nuclear.

Fraction isolation. After sacrifice, the lungs and trachea were removed intact from each animal. The lungs were lavaged with 
$0.9 \%$ saline eight times with a recovered volume of about $1500 \mathrm{ml}$. The entire lung was homogenized with an Ultra-Turrex Homogenizer in an equal volume of $0.32 \mathrm{M}$ sucrose/Tris $\mathrm{HCl}$ buffer at $\mathrm{pH} 7.4(10,13)$. Three samples of the homogenate were retained as representative of the lung parenchyma. Three $40 \mathrm{ml}$ samples of homogenate were used for isolation of lamellar body and microsome fractions via a series of differential centrifugation and sucrose step gradient ultracentrifugation procedures $(10,13)$. The lamellar bodies from the sucrose step gradients were diluted to approximately $0.2 \mathrm{M}$ sucrose and recovered as a pellet after centrifugation at $8000 \mathrm{Xg}$ for $20 \mathrm{~min}$. The lamellar body isolation procedure has been previously verified for newborn lamb lung by electron microscopy and marker enzyme analyses (13).

To demonstrate the adequacy of the alveolar wash procedure, a control study was done in which $1 \mu \mathrm{Ci}$ of $\left[{ }^{3} \mathrm{H}\right]$-labeled natural surfactant was instilled into the tracheas of twin lambs, as described above, followed by immediate sacrifice of the lambs. The lungs were removed and immediately lavaged with saline. The efficacy of the lavage procedure was estimated by comparing the amount of isotope recovered in the alveolar wash with the amount of isotope recovered in the lung homogenate fractions.

Pulmonary alveolar macrophages were recovered from two 100 $\mathrm{ml}$ aliquots of alveolar wash by an initial centrifugation at $500 \mathrm{X}$ $g$ for $20 \mathrm{~min}$ at $4^{\circ} \mathrm{C}$. The resulting pellets were resuspended in physiologic saline, and layered over $0.7 \mathrm{M}$ sucrose in saline for centrifugation at $2000 \mathrm{Xg}$ for $20 \mathrm{~min}$. The pellet was resuspended in $5 \mathrm{ml}$ normal saline. An aliquot of the resuspended pellet was taken to assess total isotope present and the rest was saved for lipid analysis.

Total $\left[{ }^{14} \mathrm{C}\right]$ radioactivity was measured in Aquasol scintillation fluid (New England Nuclear), in lung homogenate, alveolar wash, and macrophages.

Lipid analysis. Phosphatidycholine was isolated from lipid extracts (2) of the lung parenchyma, alveolar wash, lamellar bodies, microsomes, macrophages, and labeled natural surfactant by thin layer chromatography, and recovered by filtration (11). Aliquots of the phosphatidylcholine were used to measure phospholipid phosphate (1) and to measure radioactivity $\left(\left[{ }^{3} \mathrm{H}\right]\right.$ and $\left.\left[{ }^{14} \mathrm{C}\right]\right)$ in Aquasol scintillation fluid. Specific activities were determined for each sample as cpm/ $\mu$ mole phosphatidylcholine. As the kinetics of secretion of phosphatidylcholine and saturated phosphatidylcholine were similar in the newborn lamb (13), total phosphatidylcholine was studied instead of saturated phosphatidylcholine.

Data analysis. Each of the newborn lambs received both $\left[{ }^{3} \mathrm{H}\right]-$ palmitate labeled natural surfactant, and free $\left[{ }^{14} \mathrm{C}\right]$-choline, thus generating two sets of data. Each sample was studied in triplicate; thus, each data point is a mean of three values. Subcellular organelles were not isolated from two animals.

Specific activities vary with the absolute amount of isotope given to each animal and with the size of the animal. Therefore, all specific activities are normalized to $2 \times 10^{6} \mathrm{cpm}$ of administered $\left[{ }^{3} \mathrm{H}\right]$ surfactant-associated phosphatidycholine and $2 \times 10^{6} \mathrm{cpm}$ of administered $\left[{ }^{14} \mathrm{C}\right]$-choline and to a dry weight of $4.0 \mathrm{~kg}$ (e.g., if $1.9 \times 10^{6} \mathrm{cpm}$ of labeled phosphatidylcholine were given to a $3 \mathrm{~kg}$ animal, specific activities would be multiplied by $2 / 1.9 \times 3 / 4)$. To show concentration or lack of concentration of label in subcellular organelles, the specific activities of microsomes and lamellar bodies are expressed relative to the specific activities of the lung homogenate.

\section{RESULTS}

Animals. Mean pH of umbilical venous blood at the time of delivery of the lambs was $7.32 \pm 0.02$ (S.E.); mean $\mathrm{Po}_{2}$ was 32.9 \pm 3.6 torr; and mean $\mathrm{PCO}_{2}$ was $41.9 \pm 2.6$ torr. All animals appeared clinically healthy following delivery. Mean dry weight at the time of sacrifice was $4.0 \pm 0.2 \mathrm{~kg}$.

Phospholipid pool size. The mean amount of surfactant-associ-

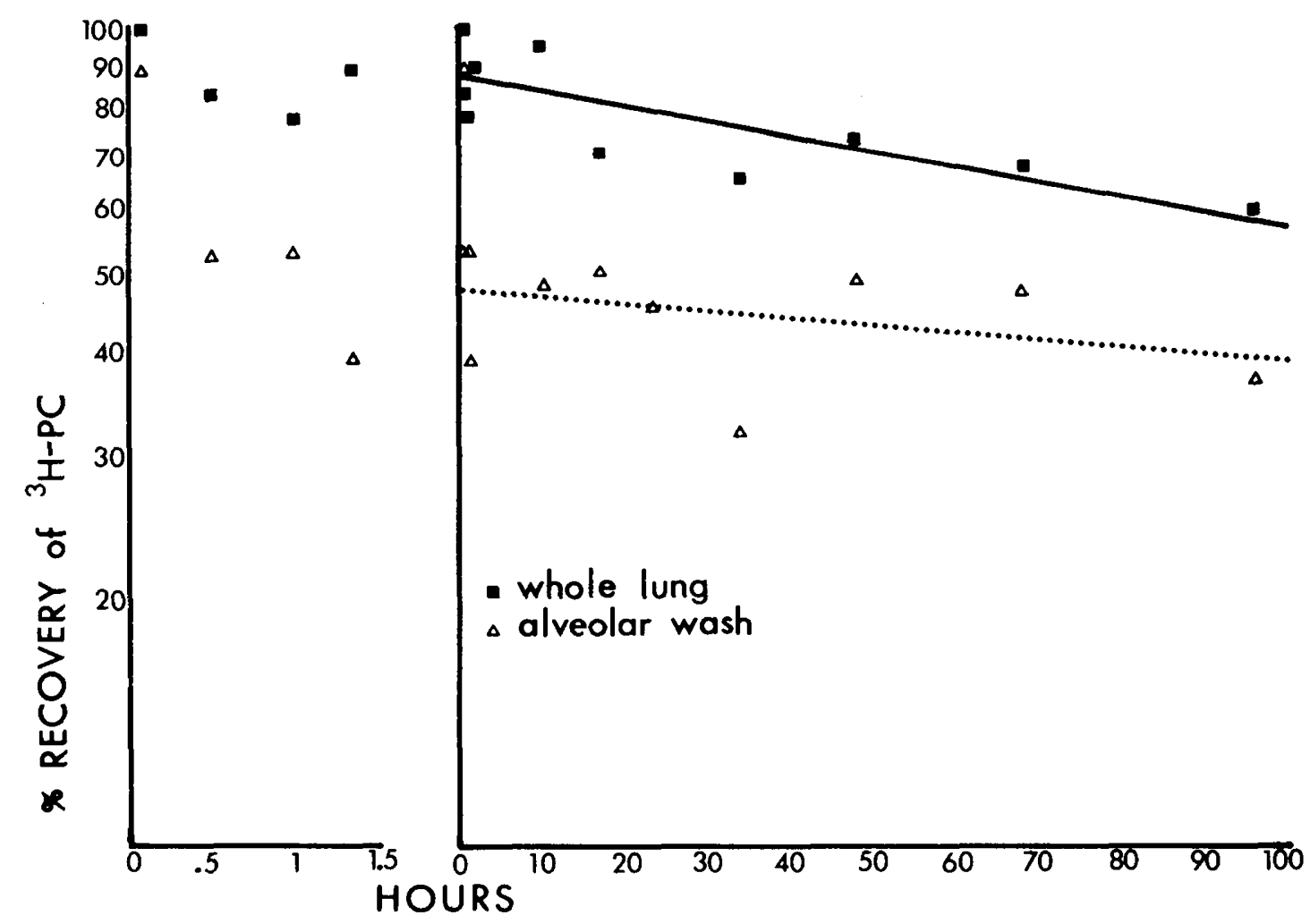

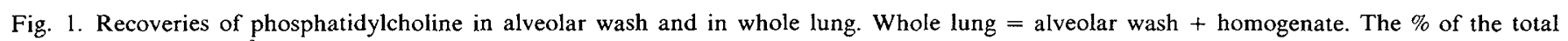

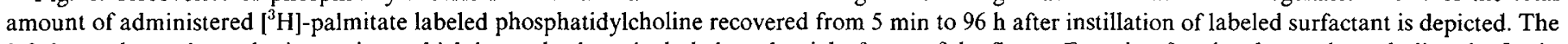

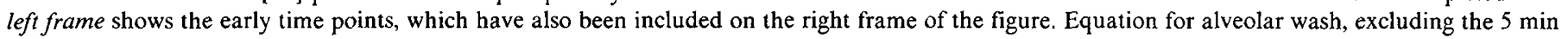

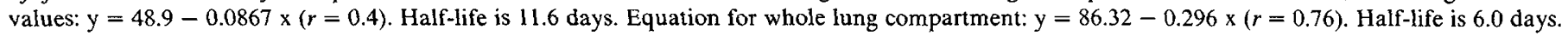
These curves are not significantly different from each other, and in fact, the half-lives could be identical. 


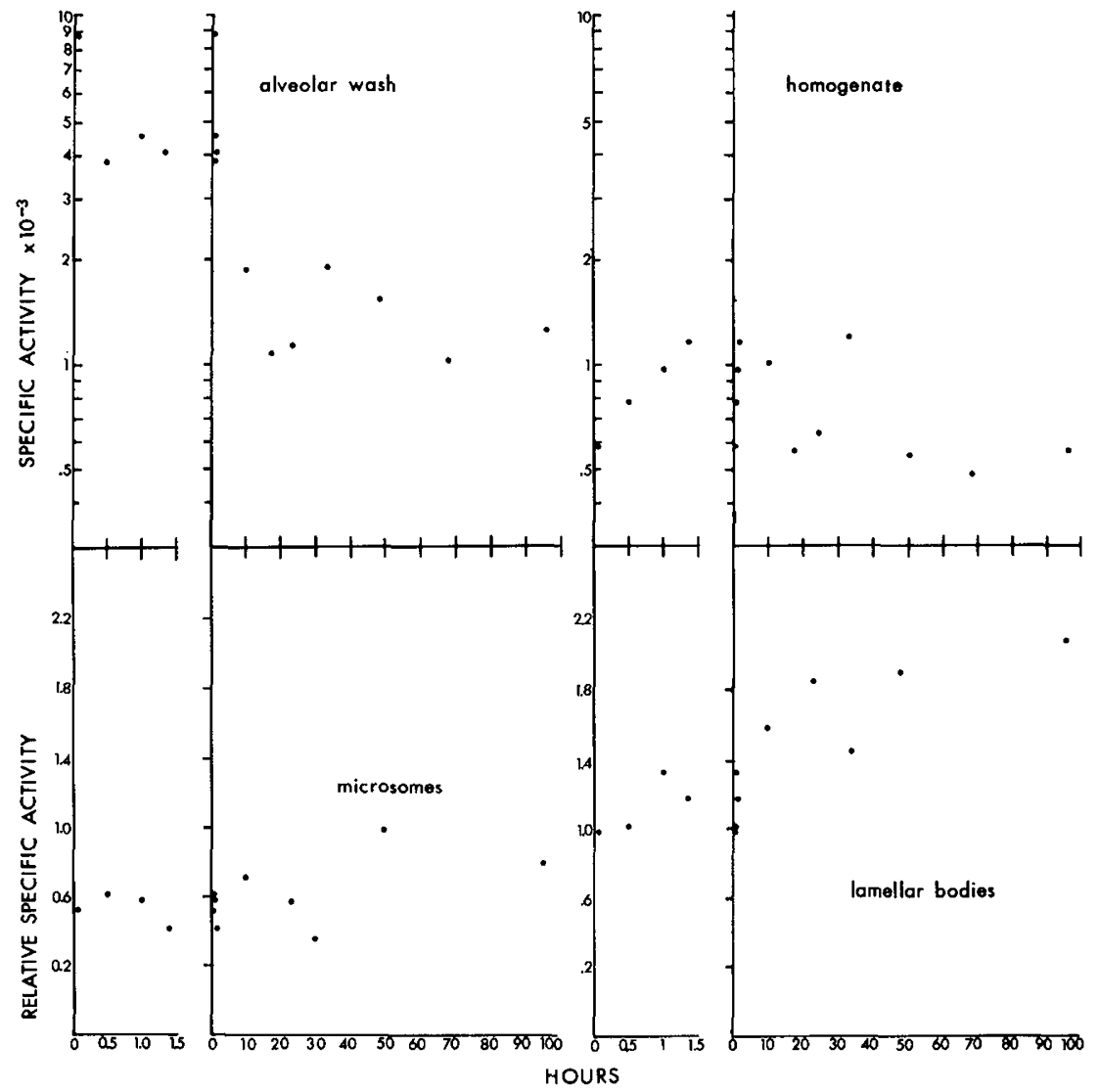

Fig. 2. Specific activities of $\left[{ }^{3} \mathrm{H}\right]$-phosphatidylcholine in lung fractions. Alveolar wash and homogenate specific activities are presented as actual specific activities. Lamellar body and microsome specific activities are presented as relative specific activities (actual specific activity $\div$ specific activity of homogenate at the same, time) to demonstrate any concentration of isotope. The initial time points are shown on an expanded time scale to the left of the time axis from $0-100 \mathrm{~h}$.

ated phosphatidylcholine given to the lambs at birth was $26.4 \pm$ $3.6 \mu$ moles $/ \mathrm{kg}$. The mean amount of phosphatidylcholine recovered in the alveolar washes was $127 \pm 19 \mu$ moles $/ \mathrm{kg}$, and the mean amount recovered in the lung homogenates was $198 \pm 24$ $\mu$ moles $/ \mathrm{kg}$. Although the dose given was not small, it represented only about $20 \%$ of the mean alveolar wash pool size and $13 \%$ of the homogenate pool size in these animals.

Disappearance of $\left[{ }^{3} \mathrm{H}\right]$-phosphatidylcholine from alveolar wash and lung tissue. Figure 1 shows the disappearance from alveolar wash and from the total lung compartment (alveolar wash and homogenate) of the $\left[{ }^{3} \mathrm{H}\right]$ labeled natural surfactant phosphatidylcholine, which was mixed with the fetal lung fluid at birth. Initially, $90 \%$ of the administered labeled phosphatidylcholine was recoverable in the alveolar wash. The slopes of the decay curves provide estimates of the biologic half-lives of phosphatidylcholine in the alveolar wash and whole lung of 11.6 and 6.0 days, respectively. The accuracy of these estimates is limited because the length of the study is short relative to the half-life values; however, the data adequately demonstrate an exceptionally long apparent half-life of alveolar surfactant in newborn lambs. The $\%$ of labeled phosphatidylcholine in the homogenate fraction was $20-50 \%$ of that administered. It is assumed to be associated with the lung tissue. The lavage procedure is effective in removing almost all recoverable phospholipid from the alveoli of newborn lambs (3).

Specific activities of $\left[{ }^{3} \mathrm{H}\right]$-phosphatidylcholine in alveolar wash and homogenate. The specific activities of $\left[{ }^{3} \mathrm{H}\right]-$ phosphatidylcholine in the alveolar wash and homogenate are shown in Figure 2. The specific activity in the alveolar wash was almost $9000 \mathrm{cpm} /$ $\mu$ mole phosphate in the animal sacrificed $5 \mathrm{~min}$ after labeled surfactant administration. This was followed by a sharp decline in the first $\mathrm{h}$ after administration, then a more gradual decline from $10 \mathrm{~h}$ to the end of the study period. The specific activity in the homogenate initially rose after isotope administration, with the value at $80 \mathrm{~min}$ approximately twice the value at $5 \mathrm{~min}$. From the specific activity at $10 \mathrm{~h}$, there was a gradual downward trend.

The specific activities of the microsomal and lamellar body fractions, relative to the specific activities of the lung homogenates, are shown in Figure 2. The relative specific activities of microsome fractions were all $<1$. Label in the lamellar bodies was more concentrated than in the surrounding homogenate with all relative specific activities $>1$ after the $30 \mathrm{~min}$ value. The specific activities of the homogenate, lamellar body, and microsomal fractions of the two animals sacrificed immediately after isotope administration were $12 \%, 13 \%$ and $10 \%$ respectively, of the alveolar wash specific activities. This demonstrates that initially little contamination of these fractions occurs from the isotope in the alveoli.

Fate of $\left[{ }^{14} \mathrm{C}\right]$-choline. The $\%$ of total $\left[{ }^{14} \mathrm{C}\right]$-choline recovered in alveolar wash plus homogenate and the $\%$ of $\left[{ }^{14} \mathrm{C}\right]$-choline incorporated into phosphatidylcholine are shown in Table 1 . The very small amounts of $\left[{ }^{14} \mathrm{C}\right]$-choline and $\left[{ }^{14} \mathrm{C}\right]$ labeled phosphatidylcholine recovered in the alveolar wash were the most striking observations. Thirty min after administration of free $\left[{ }^{14} \mathrm{C}\right]$-choline into the fetal tracheal fluid, only $4 \%$ of the $\left[{ }^{14} \mathrm{C}\right]$ was recovered in the alveoli. Although up to $28 \%$ of the administered $\left[{ }^{14} \mathrm{Cl}\right.$-choline was incorporated into phosphatidylcholine, only a small \% of the labeled phosphatidylcholine was recovered from the alveoli.

The $\left[{ }^{14} \mathrm{C}\right]$-choline specific activities of the microsomal fractions of the lung homogenates, relative to the specific activities of the homogenates, were $>1.4$ up to $80 \mathrm{~min}$, indicating concentration of isotope in microsomes (Table 2 ). The relative specific activity of the lamellar bodies was $<1$ in every instance, indicating no particular concentration of choline-labeled phosphatidylcholine in these organelles.

Macrophages. Histologic examination of hematoxylin and eosin stains revealed that more than $90 \%$ of the ceils recovered from the 
Table 1. Recovery of $\left.I^{14} C\right]$-choline and $\left[{ }^{14} C l\right.$-phosphatidylcholine in whole lung $(H+A W)^{1}$ and alveolar wash

\section{$\%$ Recovery of adminis- tered $\left[{ }^{14} \mathrm{C}\right]$-choline}

$\%\left[{ }^{14} \mathrm{C}\right]$-choline incorporated into phosphatidylcholine

\begin{tabular}{ccccc} 
Time $(\mathbf{h})$ & $(\mathrm{H}+\mathrm{AW})$ & $\mathrm{AW}$ & $(\mathrm{H}+\mathrm{AW})$ & $\mathrm{AW}$ \\
\hline 0.08 & 100 & 72 & 7 & 2 \\
0.5 & 26 & 4 & 22 & 1 \\
1 & 16 & 4 & 6 & 1 \\
1.3 & 41 & 3 & 18 & 1 \\
10 & 38 & 3 & 20 & 1 \\
17 & 9 & 4.5 & 5.2 & 3.6 \\
23 & 42 & 15 & 28 & 10 \\
34 & 18 & 5 & 7 & 1.5 \\
48 & 8.3 & 0.2 & 7 & 3 \\
68 & 16.0 & 9.9 & 9.5 & 4.9 \\
96 & 7.7 & 0.2 & 6 & 3 \\
\hline
\end{tabular}

${ }^{1} \mathrm{H}$, homogenate and $\mathrm{AW}$, alveolar wash.

Table 2. Specific activities of $\left[{ }^{14} \mathrm{C}\right]$-choline labeled phosphatidylcholine from microsomes and lamellar bodies relative to the lung homogenate

\begin{tabular}{ccc}
\hline & \multicolumn{2}{c}{ Relative specific activities } \\
\cline { 2 - 3 } Time $(\mathbf{h})$ & Microsomes & Lamellar bodies \\
\hline 0.08 & 1.55 & 0.70 \\
0.5 & 1.93 & 0.26 \\
1 & 1.40 & 0.19 \\
1.3 & 2.32 & 0.13 \\
10 & 1.05 & 0.65 \\
23 & 1.0 & 0.92 \\
34 & 0.85 & 0.73 \\
48 & 0.88 & 0.89 \\
96 & 1.0 & 1.03 \\
\hline
\end{tabular}

alveolar wash were pulmonary alveolar macrophages. Virtually no cellular debris was present among the cells. At all times during the study $<1 \%$ of the administered $\left[{ }^{3} \mathrm{H}\right]$ labeled phosphatidylcholine in natural surfactant was recovered in the macrophages obtained from 8 alveolar washes, and $<0.2 \%$ of the $\left[{ }^{14} \mathrm{C}\right]$-choline was recovered from the macrophages. The specific activities of both $\left[{ }^{3} \mathrm{H}\right]$-phosphatidylcholine and $\left[{ }^{14} \mathrm{C}\right]$-phosphatidylcholine in the macrophages were direct reflections of the alveolar wash specific activities, being nearly identical to them in most cases.

\section{DISCUSSION}

The natural surfactant used in this study has been shown to be effective in physiologic quantities in the therapy of hyaline membrane disease in premature lambs (12). Because of this, we feel confident that a good portion of the surfactant administered in this fashion actually reached the alveoli. We did not document the distribution of exogenous surfactant in the lungs, which would be a worthwhile topic for future investigation. The beneficial effect to immature lambs is much shorter lived than the half-life derived in this study would indicate. This abbreviated response has been investigated by Ikegami et al. (7) and seems to be due to a reversible inhibition of surfactant phospholipids by a protein in the alveolar fluid in animals with severe RDS, rather than because of rapid degradation. Our data in this study are quite consistent, however, with the beneficial effect of a natural synthetic surfactant mixture used by Fujiwara et al. (4) in the treatment of RDS in humans with less severe pulmonary disease than we observed in premature sheep.

The most striking finding in this study is the prolonged apparent half-life of palmitate-labeled surfactant associated phosphatidylcholine in the newborn sheep. As stated earlier, we would not interpret these half-life values to mean anything other than the fact that the apparent half-life of pulmonary phosphatidylcholine is extremely long. The half-lives, which we measured, are based on the presence of isotope in alveolar wash and homogenate. If phosphatidylcholine is being degraded and its components resynthesized into new phosphatidylcholine, the apparent half-life will be considerably longer than the actual half-life of an individual phosphatidylcholine molecule. Our findings are clearly different from the half-life values obtained by using intravenously injected surfactant precursors in adult rats and rabbits $(10,11,19,20)$. These differences are presumably due to a different mode of administration of isotope, different levels of maturity, and species differences. Oyarzun et al. (17) reported data consistent with uptake of dipalmitoyl phosphatidylcholine (DPPC) into lung tissue. Using labeled DPPC in the form of unilamellar liposomes, they found a clearance rate of DPPC from alveoli of the adult rabbit of $7.8 \% / \mathrm{h}$ (3.1\% in those with increased ventilation). Scarpelli et al. (18) in a study in which $\left[{ }^{14} \mathrm{C}\right]$ labeled phosphatidylcholine was mixed with pulmonary fluid of fetal sheep, found an early $t_{1 / 2}$ of $15 \mathrm{~min}$ in the pulmonary fluid and a late $t_{1 / 2}$ of 57 min. Oyarzun et al. (17) studied adult rabbits and administered liposomes of DPPC that are in a different physical state than natural surfactant. The small unilamellar liposomes might be more or less readily available for clearance than the natural form of intra-alveolar surfactant. Scarpelli et al. (18) studied fetal lambs at gestations greater than 135 days. Relative pulmonary immaturity (exact gestational ages are not given) could lead to different results as well as the fact that a pure synthetic labeled phosphatidylcholine, bound to bovine serum albumin, was used.

The prolonged apparent $t_{1 / 2}$ indicates extremely slow turnover of alveolar surfactant and/or considerable retutilization of label and possibly of the entire phosphatidylcholine molecule. The fact that an appreciable amount of labeled palmitate was recovered in the lung homogenate is in itself an indication that reuptake of alveolar label into the lung tissue occurs. This is consistent with the findings of Geiger et al. (5), who detected absorption of labeled aerosolized DPPC into lung tissue (particularly type 2 pneumocytes) by examining autoradiographs of frozen sections. We feel that our data are consistent with an ongoing process of reuptake of alveolar surfactant phosphatidylcholine (or at least the labeled palmitate) and either resynthesis of new labeled phosphatidylcholine or resecretion of the same phosphatidylcholine molecules. The fact that reutilization occurs has been predicted by studies in rabbits using intravenously administered precursors of surfactant phosphatidylcholine (9).

In the present study, the specific activities of $\left[{ }^{3} \mathrm{H}\right]$-phosphatidylcholine in the subcellular organelles of the lung tissue are of interest. The ratio of microsome versus homogenate specific activity is always $<1$. The microsomal fraction is considered to be the site of synthesis of phosphatidylcholine (including nonsurfactant phosphatidylcholine). If this ratio were $>1$, we could state that labeled surfactant phosphatidylcholine was concentrated in the microsomes. We cannot claim, however, that there is a lack of concentration of labeled surfactant phosphatidylcholine in the microsomes even though the ratio is $<1.0$ because nonsurfactant phosphatidylcholine is also present in the microsomes, and this will tend to decrease the specific activity. It is possible that phosphatidylcholine is absorbed into type 2 alveolar cells intact and then resecreted. Consistent with this idea is the finding that specific activities of $\left[{ }^{3} \mathrm{H}\right]$-phosphatidylcholine in lamellar bodies versus homogenates were $>1$ after $30 \mathrm{~min}$. If the labeled palmitate were cleaved from the phosphatidylcholine molecule and then used for the synthesis of new phosphatidylcholine, we might expect a greater concentration of labeled phosphatidylcholine in the microsomes, with subsequent concentration in the lamellar bodies. This pattern is seen during de novo synthesis of phosphatidylcholine, after an intravenous injection of radiolabeled palmitic acid in lambs (13).

The initial rapid decline of specific activity of labeled phosphatidylcholine in the alveolar wash reflects dilution by the alveolar phosphatidylcholine already present, dilution by early secretion 
of phosphatidylcholine during the first hours of life, and uptake of labeled phosphatidylcholine from the alveoli into other compartments. The increase in specific activity in the homogenate over the first $80 \mathrm{~min}$ reflects the initial uptake of labeled phosphatidylcholine from the alveoli. The gradual decrease in alveolar wash and homogenate specific activities after $80 \mathrm{~min}$ reflects the remarkable conservation of total palmitate label in the pulmonary compartment.

The incorporation of a sizable \% of free alveolar $\left[{ }^{14} \mathrm{C}\right]$-choline into phosphatidylcholine indicates that choline can be absorbed from the alveoli into the lung tissue where it is used as a precursor. Only a small \% of the administered choline reappears in the alveoli as labeled phosphatidylcholine, however. The probable explanation for this is that the choline is absorbed into many cells other than type 2 cells and is used in the synthesis of membrane phosphatidylcholine. Relative specific activities indicate no concentration of $\left[{ }^{14} \mathrm{C}\right]$ labeled phosphatidylcholine in the lamellar bodies, whereas there is considerable early concentration of labeled phosphatidylcholine in the microsomes. This indicates that synthesis of labeled phosphatidylcholine is taking place, but that it is not being stored in the lamellar bodies as is surfactant phosphatidylcholine $(6,11)$.

A number of recent studies in adult animals have suggested that the pulmonary alveolar macrophage (PAM) may be of importance in the synthesis or breakdown of alveolar surfactant $(3,6,14-16)$. Our findings of very low amounts of $\left[{ }^{3} \mathrm{H}\right]$-palmitate or $\left[{ }^{14} \mathrm{C}\right]$ choline labeled phosphatidylcholine in the PAM's would seem to indicate that these functions attributed to PAM's do not take place in a quantitatively significant manner in the newborn lamb. If significant degradation of surfactant occurs in PAM's, it would have to occur rapidly and the isotope would have to be disposed of rapidly because $\left[{ }^{3} \mathrm{H}\right]$ label does not accumulate in the PAM. Because $\left[{ }^{14} \mathrm{C}\right]$ label appears in PAM's only in small quantities, absorption of free alveolar choline by PAM's does not seem to play a significant part in surfactant degradation in the newborn lamb. It appears that PAM's do not utilize alveolar choline to a significant degree for phosphatidylcholine synthesis. These findings are not necessarily indicative of the function of PAM's in surfactant metabolism in adult animals.

\section{REFERENCES AND NOTES}

1. Bartlett, G. R.: Phosphorus assay in column chromatography. J. Biol. Chem., 234: 466 (1959).
2. Bligh, E. G. and Dyer, W. J.: A rapid method of total lipid extraction and purification. Can. J. Biochem. Physiol., 37: 911 (1959).

3. Fujiwara, T., Adams, F. H., El-Salawy, A., and Sipas, S.: Alveolar and whole lung phospholipids of newborn lambs. Proc. Soc. Exp. Biol. Med., 127: 962 (1968).

4. Fujiwara, T., Chida, S., Watabe, Y., Maeta, H., Morita, T., Abe, T.: Artificial surfactant therapy in hyaline membrane disease. Lancet, $1: 55$ (1980).

5. Geiger, K., Gallagher, M., and Hedley-White, J.: Cellular distribution and clearance of aerosolized dipalmitoyl lecithin. J. Appl. Physiol., 39: 759 (1975)

6. Gil, J. and Reiss, O. K.: Isolation and characterization of lamellar bodies and tubular myelin from rat lung homogenates. J. Cell. Biol., 58: 152 (1973).

7. Ikegami, M., Jobe, A., and Glatz, T.: Surfactant activity following natural surfactant (NS) treatment of premature lambs. J. Appl. Physiol., 51(2): 306 (1981).

8. Ikegami, M., Jobe, A., and Nathanielsz, P.: Labeling of pulmonary saturated phosphatidylcholine (SPC) in newborn and adult sheep. Exp. Lung Res., 2: 197 (1981).

9. Jacobs, H., Jobe, A., Ikegami, M., and Jones, S.: Surfactant phosphatidylcholine source, fluxes and turnover time in 3 day old, 10 day old, and adult rabbits. $J$. Biol. Chem., 257: 1805 (1982).

10. Jobe, A.: The labeling and biological half-life of phosphatidylcholine in subcellular fractions of rabbit lung. Biochim. Biophys. Acta, 489: 440 (1977).

11. Jobe. A., Kirkpatrick, E., and Gluck, L.: Labeling of phospholipids in the surfactant and subcellular fractions of rabbit lung. J. Biol. Chem., 253: 3810 (1978).

12. Jobe, A., Ikegami, M., Glatz, T., Yoshida, Y., Diakomanolis, E., and Padbury, J.: The duration and characteristics of treatment of premature lambs with natural surfactant. J. Clin. Invest., 67: 370 (1981).

13. Jobe, A., Ikegami, M., Sarton-Miller, I., and Barajas, L.: Surfactant metabolism of newborn lamb lungs studied in vivo. J. Appl. Physiol., 49(6): 1091 (1980).

14. Mason, R. J., Huber, G., and Vaughan, M.: Synthesis of dipalmitoyl lecithin by alveolar macrophages. J. Clin. Invest., 51: 68 (1972).

15. Naimark, A.: Cellular dynamics and lipid metabolism in the lung. Fed. Proc., 32: 1967 (1973).

16. Nichols, B. A.: Normal rabbit alveolar macrophages. I. The phagocytosis of tubular myelin. J. Exp. Med., 144: 906 (1976).

17. Oyarzun, M. J., Clements, J. A., and Baritussio, A.: Ventilation enhances pulmonary alveolar clearance of radioactive dipalmitoyl phosphatidylcholine in liposomes. Am. Rev. Respir. Dis., 121: 709 (1980).

18. Scarpelli, E. M., Condorelli, S., Colacicco, G., and Cosmi, E.: Lamb fetal pulmonary fluid. II. Fate of phosphatidylcholine. Pediatr. Res., 9: 195 (1975).

19. Tierney, D. F., Clements, J. A., and Trahan, J. H.: Rates of replacement of lecithins and alveolar instability in rat lungs. Am. J. Physiol., 213:671 (1967).

20. Young, S. L. and Tierney, D. F.: Dipalmitoyl lecithin secretion and metabolism by the rat lung. Am. J. Physiol., 222: 1539 (1972).

21. Requests for reprints should be addressed to: Dr. Theodore H. Glatz, University of California Medical Center, Irvine, Department of Pediatrics, 101 City Drive South, Orange, CA 92668.

22. This research was supported by a grant from the March of Dimes-Birth Defects Foundation, and by HD-12714 and a Research Career Development Award HD-00252 to Alan Jobe.

23. Received for publication April 14, 1981.

24. Accepted for publication January 8, 1982.

\title{
ANNOUNCEMENT
}

\author{
20th Annual Meeting of the \\ Latin American Society for Pediatric Research \\ (S.L.A.I.P.) \\ "Club el Bosque" Carretera Central, Kilometro 30, Lima, Peru \\ October 3-6, 1982
}

Fur further information please contact:

Miguel A. Oliveros, M.D., President

Rio Moche 371

Pueblo Libre

Lima

Peru 